\title{
Topological and magnetic properties of the QCD vacuum probed by overlap fermions *
}

\author{
V.V. Braguta \\ IHEP, Protvino, Moscow oblast, 142284 Russia \\ ITEP, B. Cheremushkinskaya str. 25, Moscow, 117218 Russia \\ E-mail: braqutaditep.ru
}

\section{P.V. Buividovich}

ITP, University of Regensburg, Universitätsstrasse 31, D-93053 Regensburg, Germany

E-mail: pave1.buividovichephysik.uni-regensburg.de

\section{T. Kalaydzhyan ${ }^{\dagger}$}

DESY Hamburg, Theory Group, Notkestrasse 85, D-22607 Hamburg, Germany

E-mail: tigran.kalaydzhyandesy.de

\section{M.I. Polikarpov}

ITEP, B. Cheremushkinskaya str. 25, Moscow, 117218 Russia

E-mail: polykarpeitep.ru

\begin{abstract}
We study some of the local CP-odd and magnetic properties of the non-Abelian vacuum with use of overlap fermions within the quenched lattice gauge theory. Among these properties are the following: inhomogeneous spatial distribution of the topological charge density (chirality for massless fermions) in SU(2) gluodynamics (for uncooled gauge configurations the chirality is localized on low-dimensional defects with $d=2 . .3$, while a sequence of cooling steps gives rise to four-dimensional instantons and hence a four-dimensional structure of the chirality distribution); finite local fluctuations of the chirality growing with the strength of an external magnetic field; magnetization and susceptibility of the QCD vacuum in SU(3) theory; magnetic catalysis of the chiral symmetry breaking, and the electric conductivity of the QCD vacuum in strong magnetic fields.
\end{abstract}

Xth Quark Confinement and the Hadron Spectrum

8-12 October 2012

TUM Campus Garching, Munich, Germany

\footnotetext{
*The calculations were done at the GSI Batch Farm (Darmstadt) and on the BIRD farm at DESY

† Speaker.
} 


\section{Introduction}

Recently both aspects of our studies, properties of the QCD vacuum in strong magnetic fields and its non-trivial topological structure, attracted much attention in light of ongoing heavy-ion experiments. The magnitudes of the magnetic fields possibly created there are of order of $B \sim m_{\pi}^{2}$, which may change the critical temperature of the chiral transition, several electromagnetic properties of QCD and also give rise to new phenomenological effects potentially measurable experimentally. For the origin and properties of the magnetic fields in heavy-ion collisions see the review [四].

A non-trivial gluonic background may create an imbalance between numbers of left- and right-handed quarks and

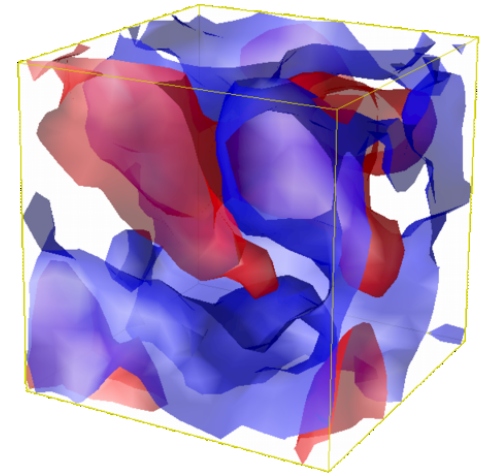

Figure 1: Isosurfaces of the chirality distribution, see [[]] for details.

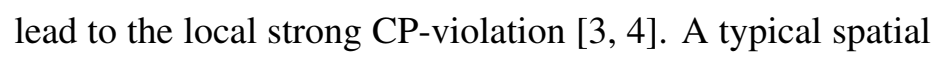
distribution of such imbalance is shown in Fig. 田, where the colors denote some fixed positive (red) and negative (blue) values of chirality. Remarkably, for uncooled gauge field configurations the distribution is irregular and, as we show later, does not follow the instanton pattern, but form low-dimensional structures.

\section{Magnetic-field-induced effects}

\subsection{Technical details}

We use a quenched $S U(3)$ lattice gauge theory with the tadpole-improved Lüscher-Weisz action []]. To generate statistically independent gauge field configurations we use the CabibboMarinari heat bath algorithm. The lattice size is $14^{4}$, and lattice spacing $a=0.105 \mathrm{fm}$. All observables we discuss later have a similar structure: $\langle\bar{\Psi} \mathscr{O} \Psi\rangle$ for VEV of a single quantity or $\left\langle\bar{\Psi} \mathscr{O}_{1} \Psi \bar{\Psi} \mathscr{O}_{2} \Psi\right\rangle$ for dispersions or correlators. Here $\mathscr{O}, \mathscr{O}_{1}, \mathscr{O}_{2}$ are some operators in spinor and color space. These expectation values can be expressed through the sum over $M$ low-lying ${ }^{1}$ but non-zero eigenvalues $i \lambda_{k}$ of the chirally invariant Dirac operator $D$ (Neuberger's overlap Dirac operator [四]:

$$
\langle\bar{\Psi} \mathscr{O} \Psi\rangle=\sum_{|k|<M} \frac{\psi_{k}^{\dagger} \mathscr{O} \psi_{k}}{i \lambda_{k}+m}, \quad \text { where } \quad D \psi_{k}=i \lambda_{k} \psi_{k}
$$

and

$$
\left\langle\bar{\Psi} \mathscr{O}_{1} \Psi \bar{\Psi} \mathscr{O}_{2} \Psi\right\rangle=\sum_{k, p} \frac{\left\langle k\left|\mathscr{O}_{1}\right| k\right\rangle\left\langle p\left|\mathscr{O}_{2}\right| p\right\rangle-\left\langle p\left|\mathscr{O}_{1}\right| k\right\rangle\left\langle k\left|\mathscr{O}_{2}\right| p\right\rangle}{\left(i \lambda_{k}+m\right)\left(i \lambda_{p}+m\right)}
$$

where all spinor and color indices are contracted and we drop them for simplicity. The uniform magnetic field $F_{12}=B_{3} \equiv B$ is introduced as described in [8]. To perform calculations in the

\footnotetext{
${ }^{1}$ We believe that the IR quantities are insensitive to the UV cutoff realized by selecting some finite number of the eigenmodes [目]
} 


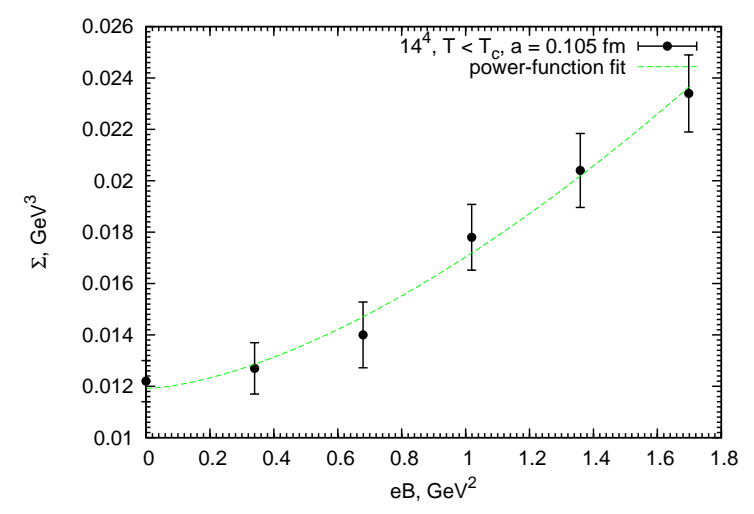

(a)

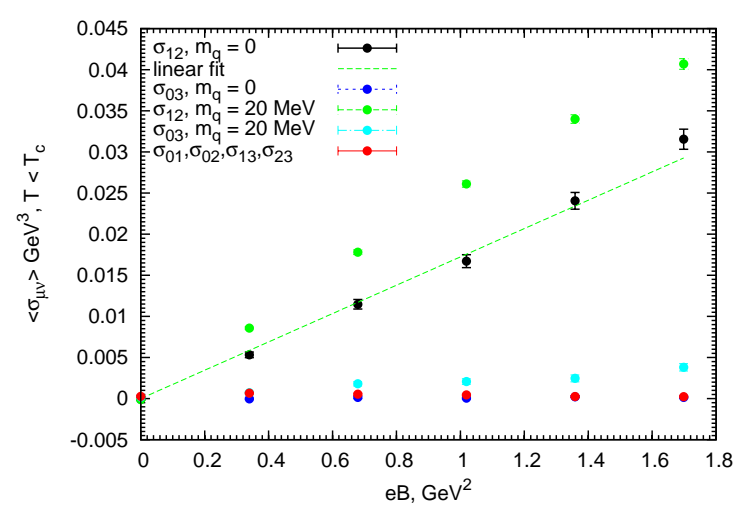

(b)

Figure 2: Chiral condensate (a) and magnetization/polarization $\left\langle\bar{\Psi} \sigma_{\alpha \beta} \Psi\right\rangle$ (b).

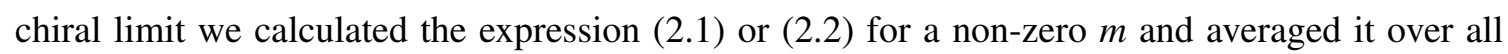
configurations of the gauge fields. Then we repeated the procedure for other quark masses $m$ and extrapolated the VEV to $m \rightarrow 0$ limit. Simulation details for the Sections 13 and $⿴ 囗 十$ are similar, but for two colors, and are described in the mentioned there papers.

\subsection{Chiral condensate}

In this section we present our results for the chiral condensate $(\mathscr{O}=\mathbb{1})$,

$$
\Sigma \equiv-\langle 0|\bar{\Psi} \Psi| 0\rangle
$$

as a function of the magnetic field $B$. The result is shown in Fig. 2(a). The general tendency for $\Sigma$ to grow with $B$ has been obtained in various models (see $[Q]$ for a review) and usually referred to the magnetic catalysis of the chiral symmetry breaking. We perform the fit of the results by the following function

$$
\Sigma^{f i t}(B)=\Sigma_{0}\left[1+\left(\frac{e B}{\Lambda_{B}^{2}}\right)^{v}\right]
$$

where $\Sigma_{0} \equiv \Sigma(0)$. The obtained fitting parameters are

$$
\Sigma_{0}=[(228 \pm 3) \mathrm{MeV}]^{3}, \quad \Lambda_{B}=(1.31 \pm 0.04) \mathrm{GeV}, \quad v=1.57 \pm 0.23 .
$$

The value of the condensate in absence of the magnetic field $\Sigma(0)$ and the exponent $v$ have reasonable values, compare with e.g. [미, ㅁ] ].

\subsection{Magnetization and susceptibility}

In this section we calculate the quantity

$$
\left\langle\bar{\Psi} \sigma_{\alpha \beta} \Psi\right\rangle=\chi(F)\langle\bar{\Psi} \Psi\rangle q F_{\alpha \beta},
$$

where $\sigma_{\alpha \beta} \equiv \frac{1}{2 i}\left[\gamma_{\alpha}, \gamma_{\beta}\right]$ and $\chi(F)$ is a coefficient of proportionality (susceptibility), which depends on the field strength. 


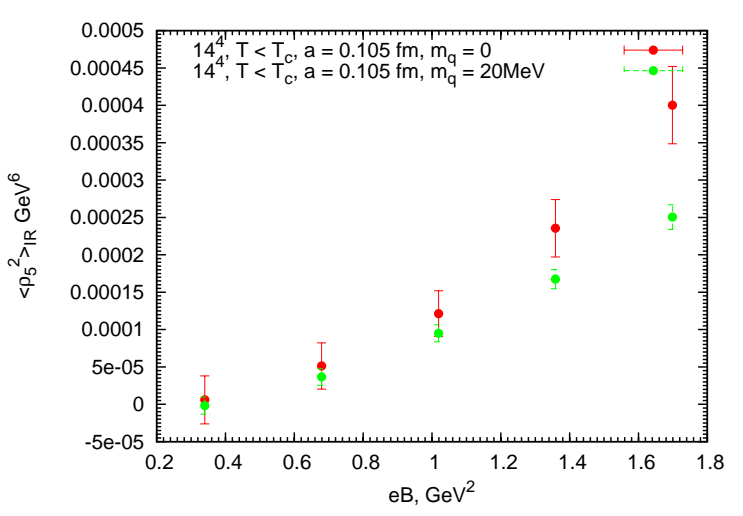

(a)

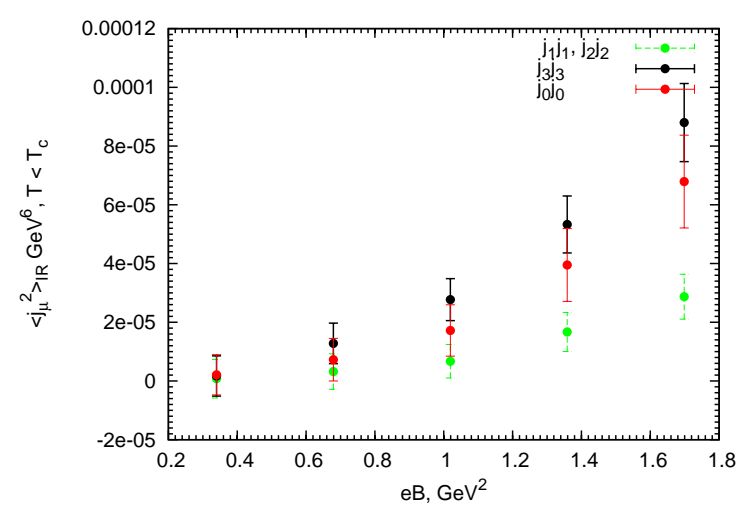

(b)

Figure 3: Fluctuations of the chirality (a) and electromagnetic current/charge (b).

This quantity was introduced in [12] and can be used to estimate the spin polarization of the quarks in external magnetic field. The magnetization can be described by the dimensionless quantity $\mu=\chi \cdot q B$, so that

$$
\left\langle\bar{\Psi} \sigma_{12} \Psi\right\rangle=\mu\langle\bar{\Psi} \Psi\rangle
$$

The expectation value (2.6) can be calculated on the lattice by (‥ is shown in Fig. 2(b). We can see, that the 12-component grows linearly with the field, which agrees with [12]. This allows us to find the chiral susceptibility $\chi(0) \equiv \chi_{0}$ from the slope of the curve. After making a linear approximation $\left\langle\bar{\Psi} \sigma_{12} \Psi\right\rangle=\Omega^{f i t} e B$, where ${ }^{2}$

$$
\Omega^{f i t} \equiv-\frac{1}{3} \chi_{0}^{f i t} \Sigma_{0}
$$

we obtain $\Omega^{f i t}=(172.3 \pm 0.5) \mathrm{MeV}$ and

$$
\chi_{0}^{f i t}=-4.24 \pm 0.18 \mathrm{GeV}^{-2}
$$

This value fits well into the range of present theoretical estimations (see [ए]3] for a review). We also have to mention a well known analytic result obtained by the OPE combined with the idea of pion dominance [14], supported by two holographic ones [15, [16], but giving a too high value comparing to our results. This disagreement seems to be an important puzzle to solve, because it will lead to a better understanding of the pion dominance assumption and the large $N_{c}$ limit.

\subsection{Evidences of the chiral magnetic effect}

One example of a new effect mentioned in the Introduction is the chiral magnetic effect (CME), which generates an electric current along the magnetic field in the presence of a nontrivial gluonic

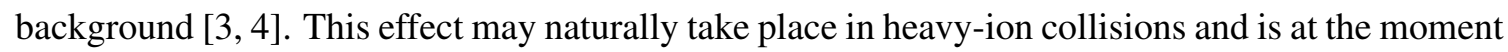
under active experimental search [127, [8, [0] (see also a review [ㅁ] on the interpretation of the

\footnotetext{
${ }^{2}$ in our simulation we calculate the magnetization of the d-quark condensate, thus $q=\left|-\frac{e}{3}\right|$
} 


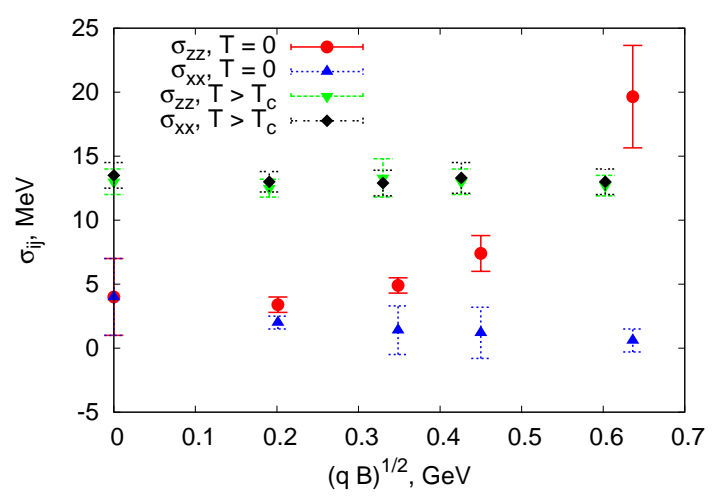

(a)

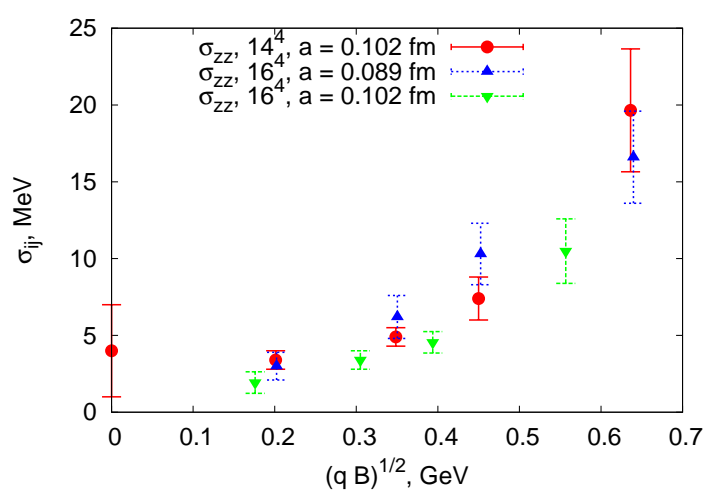

(b)

Figure 4: Conductivity at various temperatures (a) and at small temperatures and various lattices (b). Here $\sigma_{z z}\left(\sigma_{x x}, \sigma_{y y}\right)$ is the conductivity along (transverse to) the magnetic field.

experimental data). Lattice evidences of the effect can be found in [2], [23], 22], [24]. Here we implement the procedure from [2]] for the $S U(3)$ case and study the local chirality

$$
\rho_{5}(x)=\bar{\Psi}(x) \gamma_{5} \Psi(x) \equiv \rho_{L}(x)-\rho_{R}(x)
$$

and the electromagnetic current

$$
j_{\mu}(x)=\bar{\Psi}(x) \gamma_{\mu} \Psi(x)
$$

The expectation value of the first quantity can be computed by $(\mathbb{R}$. لd $)$ with $\mathscr{O}=\gamma_{5}$ and with $\mathscr{O}=\gamma_{\mu}$ for the second quantity. Both VEV's are zero, as expected, but the corresponding fluctuations obtained from ([2.2) are finite and grow with the field strength (see Fig. [1]). Here we use the "IR" subscript to emphasize, that we subtract from the quantity its value at $B=0$ :

$$
\langle Y\rangle_{I R}(B)=\frac{1}{V} \int_{V} d^{4} x\langle Y(x)\rangle_{B}-\frac{1}{V} \int_{V} d^{4} x\langle Y(x)\rangle_{B=0}
$$

One can interpret the enhancement of the current fluctuations either as short-living quantum fluctuations or as a charge flow. We measured the conductivity of the vacuum to argue in support of the latter.

\section{Conductivity of the $Q C D$ vacuum}

Electric conductivity can be extracted from the correlator of two vector currents,

$$
G_{i j}(\tau)=\int d^{3} \vec{x}\left\langle j_{i}(\overrightarrow{0}, 0) j_{j}(\vec{x}, \tau)\right\rangle
$$

Following [ [25], let us define the spectral function $\rho(w)$ which corresponds to the correlator (B.D.

$$
G_{i j}(\tau)=\int_{0}^{+\infty} \frac{d w}{2 \pi} K(w, \tau) \rho_{i j}(w), \quad \text { with } \quad K(w, \tau)=\frac{w}{2 T} \frac{\cosh \left(w\left(\tau-\frac{1}{2 T}\right)\right)}{\sinh \left(\frac{w}{2 T}\right)},
$$



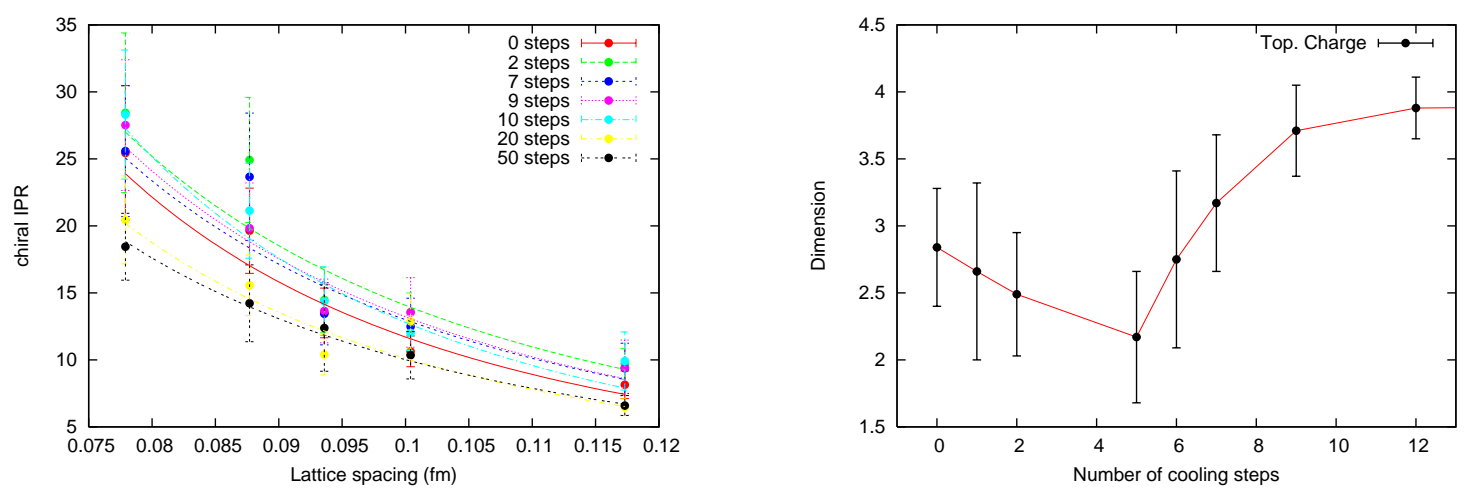

Figure 5: Chiral IPR (defined in the text) as a function of lattice spacing (a), and the dimensionality of the chirality distribution at various cooling stages (b).

where $T$ is the temperature. The Kubo formula for the electric conductivity then reads [26, [25]

$$
\sigma_{i j}=\lim _{\omega \rightarrow 0} \frac{\rho_{i j}(\omega)}{4 T} .
$$

In the limit of the weak time-independent electric field $E_{k}$, one has $\left\langle j_{i}\right\rangle=\sigma_{i k} E_{k}$. We performed the $\mathrm{SU}(2)$ quenched lattice computations (for details see [[23]) and obtained results shown in Fig. 6 . One can conclude from the results, that the QCD vacuum is an isotropic conductor in the deconfinement phase, and the conductivity does not depend on the strength of the magnetic field $\left(\sigma=15 \pm 2 \mathrm{MeV}\right.$ for $\left.T=350 \mathrm{Mev}>T_{c}\right)$. In the confinement phase the conductivity in the transverse directions is zero within error range, while it is a growing function of B-field in the longitudinal direction. In other words, the strong magnetic field at small temperatures turns the QCD vacuum from an insulator to an anisotropic conductor.

Such a behavior may also lead to the enhancement in the soft photon and dilepton production rates in the direction transverse to the magnetic field [2]].

\section{Fractal dimension of the chirality distribution}

We studied some properties of the spatial distribution of chirality (2.10), such that the volume occupied by the distribution and the fractal dimension of that volume, for various lattice spacings and cooling stages. To measure the former we used the inverse participation ratio (IPR),

$$
\operatorname{IPR}_{0}^{5}=N\left[\frac{\sum_{x}\left|\rho_{5}(x)\right|^{2}}{\left(\sum_{x}\left|\rho_{5}(x)\right|\right)^{2}}\right]_{\lambda=0} \equiv \frac{V_{\text {lattice }}}{V_{\text {distribution }}},
$$

where $N$ is the total number of sites of the lattice and the brackets $[\ldots]_{\lambda=0}$ denote an averaging over all zero modes and further averaging over all gauge field configurations. In general the IPR is equal to the inverse fraction of sites occupied by the support of a distribution. The fractal (Hausdorff) dimension $d$ can be extracted from the IPRs at various lattice spacings $a$,

$$
\operatorname{IPR}_{0}^{5}(a)=\frac{c}{a^{d}} .
$$


Lattice parameters and simulation details can be found in [[]]. The results are shown in Fig. [1]. Our conclusion is that the chirality distribution (and hence fermionic zero modes) has zero volume in the continuum limit (since IPR grows with decreasing $a$ ) and its fractal dimension equals $d=2 . .3$ for uncooled configurations (in agreement with [ [27, [28, [29, [3]]), tending to $d=4$ after the cooling. This behavior supports the idea of [B] $]$, stating that the Yang-Mills vacuum structure is different for different resolutions of the measuring procedure. The low dimensional structure of the vacuum, if true beyond the probe quark limit, may lead to a new phenomenology relevant for the heavy-ion experiments [B2], [33], B34, [35].

\section{References}

[1] K. Tuchin, arXiv:1301.0099 [hep-ph].

[2] P. V. Buividovich, T. Kalaydzhyan and M. I. Polikarpov, Phys. Rev. D 86 (2012) 074511 [arXiv:1111.6733 [hep-lat]].

[3] D. E. Kharzeev, L. D. McLerran and H. J. Warringa, Nucl. Phys. A 803 (2008) 227 [arXiv:0711.0950 [hep-ph]].

[4] K. Fukushima, D. E. Kharzeev and H. J. Warringa, Phys. Rev. D 78, 074033 (2008) [arXiv:0808.3382 [hep-ph]];

D. E. Kharzeev, L. D. McLerran and H. J. Warringa, Nucl. Phys. A 803 (2008) 227 [arXiv:0711.0950 [hep-ph]].

[5] M. Luscher and P. Weisz, Commun. Math. Phys. 97, 59 (1985) [Erratum-ibid. 98, 433 (1985)].

[6] T. A. DeGrand and A. Hasenfratz, Phys. Rev. D 64 (2001) 034512 [arXiv:hep-lat/0012021].

[7] H. Neuberger, Phys. Lett. B 417 (1998) 141 [arXiv:hep-lat/9707022].

[8] P. V. Buividovich, M. N. Chernodub, E. V. Luschevskaya and M. I. Polikarpov, Phys. Lett. B 682 (2010) 484 [arXiv:0812.1740 [hep-lat]].

[9] I. A. Shovkovy, "Magnetic Catalysis: A Review," arXiv:1207.5081 [hep-ph].

[10] P. Colangelo and A. Khodjamirian, arXiv:hep-ph/0010175.

[11] M. D'Elia and F. Negro, Phys. Rev. D 83 (2011) 114028 [arXiv:1103.2080 [hep-lat]].

[12] B. L. Ioffe and A. V. Smilga, Nucl. Phys. B 232, 109 (1984).

[13] M. Frasca and M. Ruggieri, Phys. Rev. D 83 (2011) 094024 [arXiv:1103.1194 [hep-ph]].

[14] A. Vainshtein, Phys. Lett. B 569, 187 (2003) [arXiv:hep-ph/0212231].

[15] A. Gorsky and A. Krikun, Phys. Rev. D 79 (2009) 086015 [arXiv:0902.1832 [hep-ph]].

[16] D. T. Son and N. Yamamoto, arXiv:1010.0718 [hep-ph].

[17] B. I. Abelev et al. [STAR Collaboration], Phys. Rev. Lett. 103, 251601 (2009). B. I. Abelev et al. [STAR Collaboration], Phys. Rev. C 81, 054908 (2010).

[18] N. N. Ajitanand, S. Esumi, R. A. Lacey [PHENIX Collaboration], "P- and CP-odd effects in hot and dense matter", in: Proc. of the RBRC Workshops, vol. 96, 2010.

[19] B. Abelev et al. [ALICE Collaboration], arXiv:1207.0900 [nucl-ex].

[20] A. Bzdak, V. Koch and J. Liao, arXiv:1207.7327 [nucl-th]. 
[21] P. V. Buividovich, M. N. Chernodub, E. V. Luschevskaya and M. I. Polikarpov, Phys. Rev. D 80 (2009) 054503 [arXiv:0907.0494 [hep-lat]].

[22] P. V. Buividovich and M. I. Polikarpov, Phys. Rev. D 83 (2011) 094508 [arXiv:1011.3001 [hep-lat]]; M. I. Polikarpov, O. V. Larina, P. V. Buividovich, M. N. Chernodub, T. K. Kalaydzhyan, D. E. Kharzeev and E. V. Luschevskaya, AIP Conf. Proc. 1343 (2011) 630.

[23] P. V. Buividovich, M. N. Chernodub, D. E. Kharzeev, T. Kalaydzhyan, E. V. Luschevskaya and M. I. Polikarpov, Phys. Rev. Lett. 105 (2010) 132001 [arXiv:1003.2180 [hep-lat]].

[24] A. Yamamoto, Phys. Rev. Lett. 107 (2011) 031601. [arXiv:1105.0385 [hep-lat]]; Phys. Rev. D 84 (2011) 114504. [arXiv:1111.4681 [hep-lat]].

[25] G. Aarts, C. Allton, J. Foley, S. Hands and S. Kim, Phys. Rev. Lett. 99, 022002 (2007).

[26] L. P. Kadanoff, P. C. Martin, Ann. Phys. 24, 419 (1963).

[27] I. Horvath, S. J. Dong, T. Draper, K. F. Liu, N. Mathur, F. X. Lee, H. B. Thacker, J. B. Zhang, [hep-lat/0212013].

A. Alexandru, I. Horvath, J. -b. Zhang, Phys. Rev. D72 (2005) 034506. [hep-lat/0506018].

I. Horvath, S. J. Dong, T. Draper, F. X. Lee, K. F. Liu, N. Mathur, H. B. Thacker, J. B. Zhang, Phys. Rev. D68 (2003) 114505. [hep-lat/0302009].

I. Horvath, S. J. Dong, T. Draper, F. X. Lee, K. F. Liu, N. Mathur, J. B. Zhang, H. B. Thacker, Nucl. Phys. Proc. Suppl. 129 (2004) 677-679. [hep-lat/0308029].

I. Horvath, Nucl. Phys. B710 (2005) 464-484. [hep-lat/0410046].

I. Horvath, A. Alexandru, J. B. Zhang, Y. Chen, S. J. Dong, T. Draper, F. X. Lee, K. F. Liu et al., Phys. Lett. B612 (2005) 21-28. [hep-lat/0501025].

[28] A. V. Kovalenko, S. M. Morozov, M. I. Polikarpov, V. I. Zakharov, Phys. Lett. B648 (2007) 383-387. [hep-lat/0512036].

[29] E. -M. Ilgenfritz, K. Koller, Y. Koma, G. Schierholz, T. Streuer, V. Weinberg, Phys. Rev. D76 (2007) 034506. [arXiv:0705.0018 [hep-lat]].

[30] E. -M. Ilgenfritz, K. Koller, Y. Koma, G. Schierholz, V. Weinberg, [arXiv:0912.2281 [hep-lat]].

[31] V. I. Zakharov, [hep-ph/0602141].

V. I. Zakharov, [hep-ph/0612341].

[32] T. Kalaydzhyan, arXiv:1208.0012 [hep-ph].

[33] V. I. Zakharov, arXiv:1210.2186 [hep-ph].

[34] M. N. Chernodub, J. Van Doorsselaere, T. Kalaydzhyan and H. Verschelde, arXiv:1212.3168 [hep-ph].

[35] A. R. Zhitnitsky, arXiv:1208.2697 [hep-ph]. 